\title{
Homicidios intencionales en la salud. Un tema huérfano de la seguridad asistencial
}

\author{
Intentional homicides in health. An ignored topic in healthcare security \\ Homicídios dolosos em saúde. Uma questão órfã de segurança de saúde
}

Carlos Vivas ${ }^{1}$, Homero Bagnulo²

\section{Resumen}

Los homicidios intencionales cometidos por los trabajadores sanitarios durante el desempeño de sus tareas son eventos muy poco frecuentes. Su importancia reside en que a través de su análisis se exponen las debilidades estructurales y funcionales de los sistemas de salud. Sus efectos nocivos no se limitan a las víctimas y sus familias, sino que resulta dañada la confiabilidad del sistema sanitario en su conjunto. El presente artículo resume las características de estos ataques, haciendo énfasis en las estrategias de la seguridad asistencial que pueden interceptar a los ofensores.

Palabras clave: Asesinato en serie sanitario Homicidio

Personal de salud

Daño intencional

Seguridad del paciente

Medidas de seguridad

Key words: Healthcare serial murder

Homicide

Health personnel

Intentional damage

Patient safety

Security measures

\section{Introducción}

Si un clínico decide abordar los homicidios intencionales cometidos en entornos asistenciales por el personal de la salud es probable que enfrente el desinterés, el desdén y el desagrado que el tema despierta en sus colegas. A ello debe agregarle la mirada de conmiseración que le dedicarán los actores judiciales. No obstante estas dificultades, es imprescindible que los trabajadores sanitarios se impliquen en el desarrollo de barreras que protejan a los pacientes. Después de todo, los homicidios intencionales de pacientes son el ejemplo extremo de los comportamientos disruptivos agresivos y, por tanto, es uno de los temas centrales de la seguridad asistencial.

Por homicidas seriales en la salud (health care serial killers, HCSK) se hace referencia a profesionales sanitarios que cometen homicidios intencionales de dos o más de sus pacientes en diferentes ataques, siempre actuando en entornos asistenciales y excluyendo de su motivación el móvil de piedad, eutanasia o de suicidio médicamente asistido $^{(1)}$. Esta definición es necesaria para facilitar su registro y rápida identificación en una base de datos. Sólo así será posible un seguimiento geográfico y cronológico que informe sobre las características de estos ataques.

Un segundo aspecto a destacar es la necesidad de desprenderse del modelo del asesino serial que han construido la literatura y los medios. Para ello es preciso comprender qué es el "juego de la confianza" (2). Presentarse como "gente de confianza" le permite al agresor ser aceptado por sus jefes, compañeros y, fundamentalmente, por sus pacientes. Con ello adquiere una imagen solidaria y confiable que le facilita seleccionar a su víctima,

1. Cirujano Coloproctólogo del Servicio Médico Integral. Magister en Dirección de Empresas de Salud. Fulbright Scholar. Ex Miembro de la Comisión Nacional de Seguridad del Paciente, Ministerio de Salud.

2. Ex Director del Centro de Cuidados Intensivos del Hospital Maciel. Ex Presidente del Fondo Nacional de Recursos. Ex Presidente de la Comisión Nacional de Seguridad del Paciente, Ministerio de Salud.

Los autores no han recibido ningún tipo de subvenciones para la redacción del manuscrito.

Los autores no tienen de conflicto de interés.

Correspondencia: Dr. Carlos Vivas. Correo electrónico: carlosvivasespen@gmail.com

Recibido: 18/11/2020

Aprobado: 24/3/2021

Attribution-NonCommercial 4.0 International (CC BY-NC 4.0) 
perfeccionar su método de ataque y preparar su cobertura.

La escasa producción académica sobre los HCSK se centra en el análisis post facto de la descripción del agresor, de sus víctimas y de los métodos que emplea para causar la muerte, así como en las herramientas forenses para lograr la identificación y condena de los infractores. Sin embargo, y a pesar de la existencia de herramientas proactivas, es muy poco lo que se ha publicado sobre las estrategias de la seguridad asistencial para prevenir este delito.

La razón de dar la espalda a este tema se vincula al deseo que no se hagan públicas las debilidades organizacionales que favorecen estas conductas aberrantes. Apoyándose en las supuestas ventajas de la cultura de la culpa, estrategia ya de por sí inadecuada para evitar los daños involuntarios causados a los pacientes durante su atención, se prefiere adjudicar toda la responsabilidad al infractor. De ese modo se dejan incólumes los fallos del diseño de las instituciones sanitarias que favorecen estos ataques y se robustece el estilo negador y burocrático de gestión organizacional. Pero además de ver "castigado" al agresor, la gente necesita comprender cómo fue posible que un profesional sanitario asesinara a su paciente, desafiando así el principio de que toda la sociedad comparte un mismo universo moral. Aceptar que en un grupo social coexistan diferentes dimensiones de moralidad es inquietante y, por ende, se exige una explicación que restaure el orden. Es así que a través de este relato sesgado de los factores favorecedores de estos ataques se propone a la sociedad que una vez se haya anulado al infractor será posible recuperar la confianza en el sistema. Aunque las debilidades de la institución persis$\tan$.

\section{Frecuencia}

Aunque se han publicado artículos sobre este tema desde fines del siglo XVIII, no es sino desde 1985 que comenzaron los artículos que buscan superar los relatos anecdóticos. Desde 1970 hasta 2010 la literatura informa de 90 profesionales sanitarios que fueron hallados culpables de los homicidios intencionales de sus pacientes $^{(3)}$. No hay dudas de la baja incidencia de estos delitos, aunque están lejos de tener un mero valor anecdótico. Los investigadores coinciden en que no es necesario recurrir al miedo para poner el tema en discusión. Resultaría muy fácil señalar que en 40 años "sólo" hubo 90 agresores, porque fueron los únicos casos en que se pudo probar los delitos, pero esta estrategia no es ética pues lleva al pánico y al aumento de la desconfianza en los trabajadores sanitarios. No obstante el pequeño número de agresores, se debe tener presente que los homicidios intencionales de los pacientes integran el grupo de los "eventos criminales" que publica anualmente la Joint Commission. Junto a las violaciones, hurtos, y secuestros de niños, los homicidios intencionales se mantienen desde 2014 en el $4^{\circ}$ lugar en el registro anual de los eventos centinelas de la agencia mencionada, lo que atestigua el creciente problema de la violencia en los entornos asistenciales que amenaza tanto a pacientes como a trabajadores ${ }^{(4)}$.

Además de los 90 casos señalados, se conocen 41 casos de acusados de atacar a sus pacientes que por múltiples razones no fueron hallados culpables. No obstante esto, no dejó de resultar llamativo que en la mayoría de los centros asistenciales donde se desempeñaban los acusados disminuyó el número de muertes sospechosas una vez se discontinuó su actuación ${ }^{(5)}$.

Las investigaciones de esos delitos debieron enfrentar múltiples dificultades que tuvieron buena parte de su origen en la actitud de las autoridades institucionales donde ocurrieron los asesinatos. Por lo habitual se negaron a colaborar con la excusa de que se estaría arriesgando el buen nombre de un profesional y el de la institución. En su afán por protegerse, cayeron en un silogismo de falsa oposición entre la seguridad de los pacientes y el prestigio de la organización, lo que en definitiva protegió al agresor y le permitió seguir actuando. Otra dificultad a destacar es que durante la investigación del caso índice surgieron sospechas de estar en presencia de una serie de asesinatos que se remontaban varios años en el tiempo. Así, en el caso de Niels Högel se logró probar el homicidio de 97 pacientes a lo largo de 6 años lo que demandó múltiples exhumaciones ${ }^{(6)}$.

El número confirmado de víctimas mortales en los últimos 50 años es 460 , mientras que las sospechas de homicidios alcanzan las 2.600. La cifra real es insegura pues solo en el caso del Dr. Harold Shipman una comisión investigadora ad hoc encontró pruebas de 215 asesinatos cometidos entre 1975 y 1998 mediante inyecciones intravenosas de narcóticos, mientras que al momento de su condena sólo se habían encontrado pruebas de 15 homicidios $^{(7)}$. Por su parte, Michael Swango fue hallado culpable de 4 muertes en EE.UU, mientras que existen dudas del número real de sus víctimas en Zimbabue ${ }^{(8)}$.

Hasta 2018 se cuentan 150 sobrevivientes de estos ataques, todos con graves secuelas. En especial los pacientes pediátricos presentan lesiones motoras, sensoriales y cognitivas, así como severas repercusiones en su salud mental ${ }^{(9)}$.

Otro criterio para evaluar la incidencia real de estas agresiones es a través del impacto que tiene para una organización conformar un equipo especializado en investigar estos delitos. Cuando los Hospitales de Veteranos en EE.UU. crearon un equipo interdisciplinario se logró 
la identificación y captura de 4 agresores entre los años 1995 y 2005. Estos datos permiten sospechar que la frecuencia real de estos delitos sea mayor que la registra$\mathrm{da}^{(10)}$.

\section{Agresores, víctimas y ataques}

El 86\% de los condenados fueron licenciadas en enfermería, $12 \%$ médicos y $2 \%$ tecnólogos. Aunque en números absolutos los médicos fueron la minoría, el número de sus víctimas es el mayor en todas las series. Si bien la mayoría de los agresores fueron mujeres, llama la atención el porcentaje de hombres entre los licenciados en enfermería (44\%) mientras que entre los licenciados en general los hombres representan únicamente el $6 \%$ del total ${ }^{(11)}$.

La mayoría de las víctimas fueron pacientes en estado crítico, ancianos o niños. Estos pacientes estaban desorientados, sedados, por lo que no pudieron defenderse. No obstante, un grupo de víctimas fueron pacientes ambulatorios con capacidad cognitiva conservada, lo que demuestra que nadie es inmune a estos ataques.

A pesar de que los hospitales siguen dominando las estadísticas, el porcentaje de homicidios en residenciales y hogares ha ido aumentando. Esto se vincula a que el modelo asistencial ha variado durante los últimos años, agregándose a los hospitales las residencias geriátricas y los propios hogares en el caso de las internaciones domiciliarias. Aún se desconoce la verdadera incidencia en estas localizaciones pues la identificación de un homicidio por parte de un profesional sanitario en mucho más difícil de demostrar en estos entornos ${ }^{(12)}$. Respecto a los hospitales, las unidades donde se producen más agresiones son los cuidados intensivos, donde tuvieron lugar el $25 \%$ de los homicidios descubiertos.

\section{Características}

Por lo habitual estas situaciones se presentan como una serie de muertes inesperadas que ocurren en rápida sucesión en una misma unidad y en un mismo turno. Las muertes son precedidas por un aumento brusco de la necesidad de maniobras de resucitación también en el mismo horario y en la misma unidad. Los ataques predominan en los turnos vespertinos y nocturnos, teniendo una máxima incidencia durante los cambios de turno de enfermería. Casi siempre el agresor está presente y suele participar activamente en las maniobras de resucitación e incluso iniciarlas sin dar la voz de alerta ${ }^{(13)}$. En su mayoría las muertes se producen mediante la administración intravenosa de dosis elevadas de narcóticos (morfina, fentanilo), benzodiacepinas, relajantes musculares, insulina cristalina, y soluciones hipertónicas de potasio ${ }^{(14,15)}$.

Los investigadores señalan que se trata de delitos complejos de dilucidar y que se ven confundidos por las prácticas rutinarias de un hospital que ocultan las evidencias de un acto criminal: traslado del cuerpo a la morgue, limpieza inmediata de la unidad, y el descarte tanto de las jeringas y ampollas utilizadas así como de las muestras de sangre obtenidas durante las maniobras de resucitación. A ello se debe sumar el desconocimiento que tienen los agentes judiciales respecto a la gestión clínica cotidiana, a la farmacodinamia y a los efectos secundarios de las diferentes dosis de los medicamentos empleados para causar la muerte ${ }^{(16)}$.

\section{Motivos}

El análisis de estos asesinatos está dificultado porque son muy pocos los acusados que comunican sus motivos. Tampoco ayuda la baja accesibilidad a fuentes confiables. La escasa atención que la academia presta a este tema se refleja que solo el $1 \%$ de los casos son reportados y analizados en publicaciones auditadas ${ }^{(17)}$. Por tanto, las fuentes más comunes son la prensa, las actas judiciales y los videos de algunas entrevistas a cargo de psicólogos y psiquiatras forenses. La participación activa del agresor en las maniobras de resucitación en situaciones que él mismo causa es un hecho conocido, siendo Niels Högel y Beverly Allit los casos más notorios. Esta actitud se interpreta como la búsqueda de una "ganancia secundaria" al lograr reconocimiento de sus jefes y compañeros por su excelente desempeño en situaciones de emergencia. Otras veces el motivo para asesinar parece provenir de la satisfacción sádica que deriva de poder predecir a sus colegas qué pacientes van a morir durante su guardia lo que es atribuido a su experiencia clínica $^{(18)}$.

No obstante lo señalado, es preciso establecer que la mayoría de los agresores intenta pasar desapercibido. Esto determina que las diferentes propuestas de listas de signos de detección temprana hayan fracasado. Estos signos son actitudes, afirmaciones y aspectos biográficos que aunque están correlacionados tienen un valor predictivo bajo. De todas formas, las series muestran que varios de estos signos están presentes en varios agresores: a) antecedente de fraude o de falsificación de títulos, certificados o recetas médicas; b) participación activa del ofensor en las maniobras de reanimación; c) comentarios sarcásticos sobre los fallecidos; d) anticipación de la muerte de un paciente; e) el ofensor recibe sobrenombres descalificativos de sus propios compañeros; y f) ironías respecto a estar en un período de mala suerte $^{(19)}$.

\section{Debilidades en el diseño de las instituciones asistenciales}

La ausencia de una cultura de seguridad estimula el trabajo en silos y la práctica del gradiente de autoridad mi- 
nan la transparencia y bloquean los nodos de información. La organización se niega a recibir malas noticias, tolera conductas disruptivas y tiende a negociar con el agresor la finalización del vínculo laboral. No se comunican las dudas a las autoridades sanitarias ni judiciales y tampoco se hace referencia alguna en las instancias de redactar las referencias laborales. Esta actitud negadora se prolonga en conductas revanchistas para los funcionarios que denunciaron su preocupación sobre la conducta del agresor ${ }^{(20)}$.

\section{Aportes de las políticas de seguridad asistencial}

La implementación de una cultura de la seguridad advierte a los eventuales infractores que toda vida importa y que por tanto toda muerte va a ser analizada. Ante toda muerte inesperada no se debe aceptar una actitud de resignación ${ }^{(21)}$. Del mismo modo se dejará claro que la institución no tolerará comportamientos disruptivos. Es de gran valor horizontalizar las comunicaciones intrainstitucionales aplicando estrategias de aproximación entre la gerencia y el sector operativo a través de rondas de seguridad ${ }^{(22)}$. El compromiso con las políticas de seguridad facilita la construcción de un mismo modelo mental compartido por todos los integrantes de la organización. Resulta imprescindible dar espacio a la capacidad de resiliencia de los profesionales sanitarios, siendo que la capacidad para sobreponerse a situaciones inesperadas es un activo valioso para proteger a los pacientes.

\section{Gestión del riesgo}

La seguridad asistencial dispone de varias herramientas para supervisar la calidad de los cuidados brindados a los pacientes. En primer lugar se destacan las revisiones de morbimortalidad ${ }^{(23)}$. Aunque no está diseñada para investigar un homicidio, la secuencia de la herramienta detecta el fallo de factores humanos, tecnológicos y organizacionales. Si bien la autopsia clínica es una estrategia fundamental para evaluar la calidad de la asistencia, y por tanto debiera contarse con sus aportes con mayor frecuencia, frente a toda sospecha de muerte criminal, el caso debe comunicarse al sistema legal. Por último los Global Trigger Tools, si bien son una herramienta de control administrativa, su aplicación sistemática permite objetivar el incremento de probables eventos adversos dentro de cada unidad y despertar sospechas $^{(24)}$.

La estrategia de mayor peso para interceptar los homicidios intencionales es la administración de los fármacos por pares, pues a excepción de un caso ocurrido en Austria donde 4 licenciadas actuaron en conjunto, los ofensores actúan en solitario ${ }^{(25)}$. La difícil implementación de esta medida lleva a apoyarse en la aplicación es- tricta de las medidas por la Organización Mundial de la Salud para el manejo de medicamentos peligrosos ${ }^{(26)}$. Los registros clínicos informatizados y los dispensadores inteligentes facilitan la gestión del manejo seguro de los medicamentos ${ }^{(27)}$. No obstante su utilidad, cabe recordar que varios ofensores utilizaron en su favor el conocimiento de los modos de fallas de estos dispositivos. También son de ayuda las cámaras de video vigilancia de alta definición. Resulta complejo para el infractor justificar su aproximación a un paciente cuando no fue convocado o cuando no estaba indicado realizar controles o administrar medicación.

De lo expuesto surge la necesidad por parte de los Ministerios de Salud Pública, Interior y Poder Judicial de adoptar una conducta proactiva en este tema y generar grupos de estudio para el diseño de estrategias y herramientas que permitan a la sociedad establecer una detección lo más temprana posible de estos delitos.

\section{Abstract}

Intentional homicides committed by health workers in the course of their duties are very rare events. Its importance resides in the fact that the structural and functional weaknesses of health systems are exposed through its analysis. Moreover, their harmful effects are not limited to the victims and their families, since the reliability of the health system as a whole is damaged. This article summarizes the characteristics of these attacks, emphasizing the strategies of healthcare security that can intercept offenders.

\section{Resumo}

Homicídios dolosos cometidos por profissionais de saúde no exercício de suas funções são eventos muito raros. A sua importância reside no fato de, através da sua análise, expor as fragilidades estruturais e funcionais dos sistemas de saúde. Seus efeitos nocivos não se limitam às vítimas e suas famílias, e faz com que a confiabilidade no sistema de saúde como um todo fique prejudicada. Este artigo resume as características desses ataques, enfatizando as estratégias de segurança da saúde que podem interceptar os infratores.

\section{Bibliografía}

1. Lubaszka CK, Shon PC. Reconceptualizing the notion of victim selection, risk, and offender behavior in healthcare serial murders. J Crim Psychol 2013; 3(1):65-78.

2. Lubaszka CK, Shon PC, Hinch R. Healthcare serial killers as confidence men. J Investig Psych Offender Profil 2014; $1: 1-28$.

3. The Long-Term Care Homes Public Inquiry; Yorker BC. Expert report on safety and security of residents in the long-term care homes system. Ontario, 2018. Disponible en: 
https://longtermcareinquiry.ca/en/exhibits/ [Consulta: 23 junio 2019].

4. The Joint Commission. Summary data of sentinel events reviewed. Oakbrook Terrace (IL), 2021. Disponible en: https://www.jointcommission.org/-/media/tjc/documents/resources/patient-safety-topics/sentinel-event/summary-se-report-2020.pdf [Consulta: 23 junio 2019].

5. Sackman B, Vecchione M, Shmetterer J. Behind the murder curtain. New York: Post Hill, 2018:chap 22. [Documento de Kindle].

6. Niels Högel: German ex-nurse convicted of killing 85 patients. BBC News, 6 june 2019. Disponible en: https://www.bbc.com/news/world-europe-48539894 [Consulta: 28 febrero 2020].

7. United Kingdom. Department of Health. The shipman inquiry: the final report. 27 january 2005. Disponible en: https://webarchive.nationalarchi-

ves.gov.uk/20090808155110/http://www.the-shipman-inquiry.org.uk/reports.asp [Consulta: 13 agosto 2012].

8. Wolf ZR, Donohue-Smith M, Wolf KC. Nurses who murder or who are accused of murder. Int J Hum Caring 2019; 23(1):51-70.

9. Sackman B, Vecchione M, Shmetterer J. Behind the murder courtain. New York: Post Hill, 2018:chap 9. [Documento de Kindle].

10. Sackman B. When the ICU becomes a crime scene. Crit Care Nurs Q 2015; 38(1):30-5.

11. Yorker BC, Kizer KW, Lampe P, Forrest AR, Lannan JM, Russell DA. Serial murder by healthcare professionals. J Forensic Sci 2006; 51(6):136-271.

12. Gillesse EE. Report of the Public Inquiry into the Safety and Security of Residents in the Long-Term Care Homes System. A systemic inquiry into the offences. Ontario, 2019. v.2. Disponible en: http://longtermcareinquiry.ca/wp-content/uploads/LTCI_Final_Report_Volume2_e.pdf [Consulta: 23 junio 2019].

13. Yorker BC. The legal side: nurses accused of murder. Am J Nurs 1988; 88(10):1327-32.

14. Johnstone RE, Katz RL, Stanley TH. Homicides using muscle relaxants, opioids, and anesthetic drugs: anesthesiologist assistance in their investigation and prosecution. Anesthesiology 2011; 114(3):713-6.
15. Farrell M. Health-care serial poisoning. En: Farrell M. Criminology of serial poisoners. Palgrave Macmillan, 2018:93-114.

16. Graeber C. The good nurse: a true story of medicine, madness, and murder. New York: Hachette Book Group, 2013:chap 63. [Documento de Kindle].

17. Field J, Pearson A. Caring to death: the murder of patients by nurses. Int J Nurs Pract 2010; 16:301-9.

18. Beine KH. Homicides of patients in hospitals and nursing homes: a comparative analysis of case series. Int J Law Psychiatry 2003; 26:373-86.

19. Yardley E, Wilson D. In search of the 'Angels of Death': conceptualising the contemporary nurse healthcare serial killer. J Investig Psych Offender Profil 2016; 13:39-55.

20. Henriksen K, Dayton E. Organizational silence and hidden threats to patient safety. Health Serv Res 2006; 41(4 Pt 2):1539-54.

21. Bagnulo H, Vivas C. ¿Cómo evitar que se repita? Arch Med Interna 2012; 34(3):65-6.

22. Martin G, Ozieranski P, Willars J, Charles K, Minion J, McKee L, et al. Walkrounds in practice: corrupting or enhancing a quality improvement intervention? Jt Comm J Qual Patient Saf 2014; 40(7):303-10.

23. Bal G, David S, Sellier E, François P. Intérêt des revues de mortalité et de morbidité pour la formation des médecins et l'amélioration de la qualité et de la sécurité des soins: revue de la littérature. Presse Med 2010; 39(2):161-8.

24. de Wet C, Bowie P. The preliminary development and testing of a global trigger tool to detect error and patient harm in primary-care records. Postgrad Med J 2009; 85(1002): 176-80.

25. Wolf ZR, Hughes RG. Best practices to decrease infusion-associated medication errors. J Infus Nurs 2019; 42(4):183-92.

26. World Health Organization. Medication safety in high-risk situations. Technical Report. Geneva: WHO, 2019.

27. The Long-Term Care Homes Public Inquiry; Greenall J. Expert report on safety and security of medication use process in residents in the long-term care homes system. Ontario, 2018. Disponible en: https://longtermcareinquiry.ca/en/exhibits/ [Consulta: 23 junio 2019].

\section{Contribución de autores}

Ambos autores han participado en todas las etapas de la concepción, diseño y redacción del manuscrito.

Carlos Vivas, ORCID 0000-0002-1647-1830

Homero Bagnulo, ORCID 0000-0002-7608-3899 